East African Medical Journal Vol. 86 No. 7 July 2009

USE OF INSECTICIDE TREATED BED NETS AMONG PREGNANT WOMEN IN KILIFI DISTRICT, KENYA

F.K. Njoroge, MBChB, MPH, V. N. Kimani, PhD, MA, BA, Associate Professor, D. Ongore, PhD, MPH, MBChB, Senior Lecturer, Department of Community Health, College of Health Sciences, University of Nairobi, P.O. Box 19676-0202 Nairobi and W. S. Akwale, PhD, MBChB, Head, Division of Malaria Control, Ministry of Health, P.O. Box 30016, Nairobi, Kenya

Request for reprints to: Dr. F. K. Njoroge, P.O. Box 1212, Kilifi, Kenya

\title{
USE OF INSECTICIDE TREATED BED NETS AMONG PREGNANT WOMEN IN KILIFI DISTRICT, KENYA
}

\author{
F.K. NJOROGE, V. N. KIMANI, D. ONGORE and W.S. AKWALE
}

\begin{abstract}
Background: Malaria is one of the most serious public health problems in Kenya. Pregnant women are among the groups with the highest risk of malaria. Use of insecticide treated bed nets (ITNs) is a cost-effective method of controlling malaria. Despite this, there is low utilisation of ITNs among pregnant women in Kilifi district which is an endemic malaria zone.

Objective: To determine knowledge, attitude and practice on the use of ITNs in the prevention of malaria among pregnant women in Kilifi district.

Design: A descriptive cross-sectional study.

Setting: The district hospital and the five health centres in Kilifi district

Subjects: Two hundred and twenty pregnant women attending antenatal clinics (ANC) between October and December 2007.

Results: Knowledge on malaria illness and ITNs was high with majority of pregnant women having adequate level of knowledge $(86.9 \%)$. There was significant association between level of education and adequate knowledge (P-value=0.010). Good attitude on ITNs use was low. There was no association between good attitude and any of the socio-demographic variables. The majority of pregnant women attending ANC owned ITNs $(75.4 \%)$. ITNs usage was high $(70.5 \%)$. There was significant association between religion and good practice ( $p$-value $=0.050$ ). Although adequate level of knowledge on malaria and protective role of ITNs was high, there was no association between knowledge with practice and attitude.

Conclusion: Before any malaria preventive intervention is implemented in an area, different socio-cultural factors must be considered when behavioural interventions for malaria control are designed and implemented. Targeted health education should be disseminated to the community to remove stigma and misconceptions associated with ITNs. Community concerns and fears should be addressed.
\end{abstract}

\section{INTRODUCTION}

Malaria is the leading cause of morbidity and mortality in many developing countries, where between 350 and 500 million clinical episodes of malaria occur every year. At least one million deaths occur every year due to malaria with more than $80 \%$ of the malaria deaths worldwide occurring in Africa south of the Sahara (1). Malaria infects 20 million Kenyans annually (2). In Kilifi, malaria accounts for $37 \%$ of top ten causes of morbidity in the district (3).

Malaria is transmitted via the bite of female Anopheles mosquito during a blood meal. Blood provide necessary nutrients for the development of the eggs in the ovaries (5).
The use of mosquito nets as a protection from mosquito bites during the night remains the most important of all measures of personal protection. ITNs generates a chemical halo that extends beyond the mosquito net itself. This tends to repel or deter mosquitoes from biting or shorten the mosquito's life span so that she cannot transmit malaria infection (6). ITNs prevents malaria as a result of both the personal protection and alsothe' masseffect' on thelocal mosquito population when they are used on a community-wide basis (7). ITNs are safe to use. Pyrethroids have no tendency for bioaccumulation and breakdown rapidly in mammalian tissue in the soil (6).

Several studies done in malarial zones in Africa, Asia and Latin America demonstrate that 
knowledge, attitude and practices (KAP) affects ITNs use. In Kenya, giving free ITNs to pregnant women increased ITNs use (8). A simple health promotion message raised ITNs use in remote areas of Myanmar, Southeast Asia (9).

Studies in Senegal, Nigeria, Zambia, Ghana and Ethiopia, found that, ITNs were more likely to be used than untreated ones, nets two years old or less were more likely to be used than older nets and nets that were paid for were more likely to be used than nets obtained for free (10).

ITNs were used by few people in Uganda and Western Kenya, mainly because of their high cost and the perception that the chemicals used to treat them have dangerous effects on pregnancy and the foetus $(11,12)$. In Ghana, many community members did not associate malaria with the mosquito in theory or practice (13).

In five West African countries (Ghana, Gambia, Guinea Bissau, Sierra Leone and Senegal), Guatemala, and Mexico, knowledge of the cause of malaria was low. People were more concerned about mosquitoes being a nuisance than a cause of the infection (14, $16,17)$.

In western Kenya, Burkina Faso and Australia, use of ITNs depended strongly on weather, in which ITNs were more likely to be used in periods of cooler weather and rainy seasons (18-20). The ITNs use in Australia, and Zimbabwe was also influenced by perception that mosquito density was low at home $(20,21)$

In Kilifi district, only $7.9 \%$ of pregnant women slept under ITNs (22). This coverage is very low; the area being an endemic malaria zone. The study is designed to assess the role of KAP in influencing the use of ITNs among pregnant women in Kilifi district.

\section{MATERIALS AND METHODS}

This was a descriptive cross sectional study. The study population was pregnant women attending antenatal clinic (ANC) in the district hospital and the five health centres. Probability proportion to number of attendance per year wasused to determinethesamplesizeincluded in each health facility. Simple random sampling was used to select the women attending ANC. Quantitative data were collected using a structured questionnaire administered to pregnant women. Qualitative data were obtained through focus group discussions and a key informantinterview. The data were analysed by use of SPSS computer package and the results presented in descriptive and inferential methods.

\section{RESULTS}

Socio-demographic characteristic:A total of 220 pregnant women were interviewed. Their ages ranged from 15 to 45 years with a mean age of 25 . The mean parity was Para 3 and median was 1 . Majority were married (95\%). There were three religious groups (Christians, traditionists and Muslims) with Christians being the majority (57.3\%). A big proportion of pregnant women were unemployed $(57.3 \%)$. There was a high illiteracy level. Those without any formal education were $34.1 \%$ while those with college education and above constituted only $2.7 \%$.

\section{Knowledge on malaria and ITNs}

Transmission, symptoms and complications: Malaria was reported to be the main health problem $(81.7 \%)$ of study women. Nearly all women said malaria was transmitted through mosquito bite $(98.2 \%)$ and few thought malaria was transmitted through dirty water $(1.4 \%)$. All the pregnant women knew of at least one main symptom and complication of malaria. Malaria was considered as a preventable disease in pregnancy by majority $(97.3 \%)$. This was by use of ITNs $(90.9 \%)$ and by taking of drugs / herbs in $7.4 \%$ of the women.

Preventive role of ITNs: Majority of the women (74.5\%) knew the difference between ITNs and ordinary bed. The advantage of sleeping under ITNs was to prevent malaria $(71.2 \%)$ while $(27.4 \%)$ said it prevented mosquito nuisance. Time that ITNs should be used was varied with $(85.9 \%)$ mentioning every night while some $(13.6 \%)$ mentioned during rainy seasons.

Level of knowledge: Knowledge on malaria and ITNs was determined by a scoring system. From this scoring system, $(86.9 \%)$ of pregnant women had adequate knowledge while $(12.3 \%)$ had inadequate knowledge.

Results of logistic regression analysis: Only level of education $(\mathrm{OR}=2.71,95 \% \mathrm{CI}: 0.02-0.205$, P-value $=0.045$ ) was found to be significantly related to level of knowledge. 
Table 1

Cross tabulation between socio-demographic factors and level of knowledge on malaria and ITNs

\begin{tabular}{|c|c|c|c|c|c|}
\hline \multirow[t]{3}{*}{ Variable } & \multirow{2}{*}{\multicolumn{2}{|c|}{$\begin{array}{l}\text { Inadequate } \\
\text { knowledge }\end{array}$}} & \multicolumn{2}{|c|}{ Adequate } & \multirow{3}{*}{ P-value } \\
\hline & & & knor & ledge & \\
\hline & No. & $(\%)$ & No. & $(\%)$ & \\
\hline \multicolumn{6}{|l|}{ Age (years) } \\
\hline $15-34$ & 25 & 92.6 & 176 & 91.2 & \\
\hline $35+$ & 2 & 7.4 & 17 & 8.8 & 0.570 \\
\hline \multicolumn{6}{|l|}{ Parity } \\
\hline Parous & 20 & 74.1 & 144 & 75.0 & \\
\hline Grand-multi-parous & 7 & 25.9 & 48 & 25.0 & 0.540 \\
\hline \multicolumn{6}{|l|}{ Marital status } \\
\hline Married & 27 & & 182 & 94.3 & \\
\hline Unmarried & 0 & & 11 & 5.7 & 0.230 \\
\hline \multicolumn{6}{|l|}{ Religion } \\
\hline Christian & 12 & 44.4 & 144 & 59.1 & \\
\hline Non-Christian & 15 & 55.6 & 79 & 40.9 & 0.110 \\
\hline \multicolumn{6}{|l|}{ Occupation } \\
\hline Salaried & 3 & 11.1 & 48 & 24.9 & \\
\hline Unsalaried & 24 & 88.9 & 145 & 75.1 & 0.080 \\
\hline \multicolumn{6}{|l|}{ Education } \\
\hline Formal & 12 & 44.4 & 133 & $8.9 \%)$ & \\
\hline Infomal & 15 & 55.6 & 60 & $.1 \%)$ & 0. 010 \\
\hline
\end{tabular}

There was significant association between education and adequate level of knowledge $(P$-value $=0.010)$. Only education was significantly associated with adequate level of knowledge among the socio-demographic variables.

Table 2

Regression analysis of socio-demographic variable against level of knowledge

\begin{tabular}{llllll}
\hline Variable & $\begin{array}{l}\text { B(Regression } \\
\text { coefficient) }\end{array}$ & $\begin{array}{l}\text { Wald's } \\
\text { statistics }\end{array}$ & $\begin{array}{l}\text { Odds } \\
\text { ratio }\end{array}$ & $95 \%$ CI & P-value \\
\hline Age & 0.01 & 0.011 & 1.27 & $-0.168-0.170$ & 0.991 \\
Parity & -0.026 & -0.469 & 1.05 & $-0.137-0.084$ & 0.639 \\
Marital status & 0.133 & 1.297 & - & $-0.069-0.335$ & 0.196 \\
Religion & 0.024 & 0.507 & 1.804 & $-0.07-0.118$ & 0.613 \\
Occupation & 0.053 & 0.982 & 2.648 & $-0.053-0.159$ & 0.327 \\
Education & 0.104 & 2.020 & 2.771 & $0.02-0.205$ & 0.045 \\
\hline
\end{tabular}

Attitudes on malaria

UseofITNs: Among women who did not ownITNs, the main reason was lack of money (84.6\%). Other reasons was not bothered by mosquitoes and assumption that ITNs was not important.

There was ITNs colour preference. Many had blue ITNs $(42.4 \%), 35.6 \%$ had white and $22 \%$ had green bed nets. Some women did not use ITNs the night prior to the interview. Majority (75\%) said it felt hot inside the net, some $(16 \%)$ said that there were no mosquitoes during that period and $8.3 \%$ did not have ITNs. Majority of the women $(85.9 \%)$ used rectangular shaped ITNs, few $(13.5 \%)$ used conical shape and $0.5 \%$ were not sure of the shape.

FGD revealed that in 2006, government gave out ITNs to pregnant women for free in its campaign to curb malaria. But many ITNs were returned back by the same women they were meant to benefit. This was primarily due to socio-cultural beliefs among 
Mijikenda ethnic groups. The bed nets issued were white in colour and had a rectangular shape.

Women explained that, "According to Mijikenda culture, when somebody dies, the casket which is rectangular in shape is wrapped with a white sheet as it is taken to the grave site. By sleeping under white rectangular bed nets, it resembled a dead person in coffin ready for burial." This made them uncomfortable to use bed nets. Those who attempted to sleep under them said that they experienced bad dreams. It is from these bad dreams that women said that bed nets were talking to them.

Many women were ready to use bed nets as long as they were not white and rectangular. "We prefer blue or green colour and conical shape as they are more in line with our culture".

Level of attitude on use of ITNs: Level of attitude on use of ITNs was determined by a scoring system where several questions were asked and marks awarded. A cut-off point of 50\% was put. Pregnant women who scored above 50\% were regarded as having good attitude. Those who scored less were classified as having bad attitude. From this scoring system, 22.1\% of pregnant women had a good attitude and $77.9 \%$ had a bad attitude.

Table 3

Cross tabulation between socio-demographic factors and attitude towards use of ITNs

\begin{tabular}{|c|c|c|c|c|c|}
\hline \multirow{2}{*}{$\begin{array}{l}\text { Variable } \\
\text { Age (years) }\end{array}$} & \multicolumn{2}{|c|}{$\begin{array}{l}\text { Bad attitude } \\
\text { No. }(\%)\end{array}$} & \multicolumn{2}{|c|}{$\begin{array}{l}\text { Good attitude } \\
\text { No. }(\%)\end{array}$} & P-value \\
\hline & & & & & \\
\hline $15-34$ & 157 & 91.8 & 49 & 89.8 & \\
\hline $35+$ & 14 & 8.2 & 5 & 10.2 & 0.420 \\
\hline \multicolumn{6}{|l|}{ Parity } \\
\hline Parous & 129 & 74.4 & 35 & 72.9 & \\
\hline Grand-multi-parous & 42 & 24.6 & 13 & 27.1 & 0.430 \\
\hline \multicolumn{6}{|l|}{ Marital status } \\
\hline Married & 163 & 95.3 & 46 & 93.9 & \\
\hline Unmarried & 8 & 4.7 & 3 & 6.1 & 0.460 \\
\hline \multicolumn{6}{|l|}{ Religion } \\
\hline Christian & 94 & 55.0 & 32 & 65.3 & \\
\hline Non-Christian & 77 & 45.0 & 17 & 34.7 & 0.130 \\
\hline \multicolumn{6}{|l|}{ Occupation } \\
\hline Salaried & 38 & 22.2 & 13 & 26.5 & \\
\hline Unsalaried & 133 & 77.8 & 36 & 73.5 & 0.330 \\
\hline \multicolumn{6}{|l|}{ Education } \\
\hline Formal & 110 & 64.3 & 35 & 71.4 & \\
\hline Informal & 61 & 35.7 & 14 & 28.6 & 0.230 \\
\hline
\end{tabular}

There was no significant association between any socio-demographic variable with good attitude on use of ITNs.

Results of logistic regression analysis: No socio-demographic variable was significantly related to attitude on use of ITNs.

Table 4

Regression analysis of socio-demographic variable against attitude on use of ITNs

\begin{tabular}{llllll}
\hline Variable & $\begin{array}{l}\text { B (Regression } \\
\text { coefficient) }\end{array}$ & $\begin{array}{l}\text { Wald's } \\
\text { statistics }\end{array}$ & $\begin{array}{l}\text { Odds } \\
\text { ratio }\end{array}$ & $95 \%$ CI & P- value \\
\hline Age & -0.024 & -0.215 & 1.274 & $-0.239-0.192$ & 0.830 \\
Parity & -0.043 & -0.606 & 0.877 & $-0.184-0.098$ & 0.545 \\
Marital status & 0.056 & 0.427 & 1.329 & $-0.203-0.315$ & 0.670 \\
Religion & 0.057 & 0.932 & 1.542 & $-0.063-0.177$ & 0.352 \\
Occupation & 0.029 & 0.420 & 1.264 & $-0.107-0.165$ & 0.675 \\
Education & 0.033 & 0.501 & 1.386 & $-0.617-0.165$ & 0.617 \\
\hline
\end{tabular}


Practices of ITNs use

ITNs use in prevention of malaria: Majority of pregnant women owned bed nets $(88.2 \%)$ and 11.8 did not. Many of the pregnant women used ITNs as a method of preventing malaria $(70.5 \%)$. Ordinary bed nets followed with 17.7 and 12.5 used other methods namely mosquito coil, in-house spraying, drugs (metakelfin or fansidar) and traditional herbs.

In FGD, some women had not been used to sleeping under bed nets. "Traditionally, we grew-up withoutsleeping under abed net or seeing our parents using them and as adult women; we are not used to them".
Others said that it felt hot under the bed net and therefore uncomfortable especially during the hot seasons. "During hot seasons, a bed net makes you sweat more which makes it difficult to sleep comfortably."

Few sighted sleeping pattern as a hindrance. "We sleep on the floor especially during hot seasons and not on the bed which makes it difficult to use bed nets."

Level of practice on use of ITNs in prevention of malaria during pregnancy: Level of practice on use of ITNs was determined by a scoring system. From this scoring system, 85.1 of pregnant women had good practice while 14.1 had bad practice.

Table 5

Cross tabulation between socio-demographic factors and practice of use of ITNs

\begin{tabular}{|c|c|c|c|c|c|}
\hline \multirow[t]{2}{*}{ Variable } & \multicolumn{2}{|c|}{ Bad practice } & \multicolumn{2}{|c|}{ Good practice } & \multirow[t]{2}{*}{$\mathrm{p}$-value } \\
\hline & No. & $(\%)$ & No. & $(\%)$ & \\
\hline \multicolumn{6}{|l|}{ Age (years) } \\
\hline $15-34$ & 29 & 93.5 & 172 & 91.0 & \\
\hline $35+$ & 2 & 6.5 & 17 & 9.0 & 0.480 \\
\hline \multicolumn{6}{|l|}{ Parity } \\
\hline Parous & 24 & 77.4 & 140 & 74.5 & \\
\hline Grand-multi-parous & 7 & 22.6 & 48 & 25.5 & 0.460 \\
\hline \multicolumn{6}{|l|}{ Marital status } \\
\hline Married & 28 & 90.3 & 181 & 95.8 & \\
\hline Unmarried & 3 & 9.7 & 8 & 4.2 & 0.190 \\
\hline \multicolumn{6}{|l|}{ Religion } \\
\hline Christian & 13 & 41.9 & 126 & 59.8 & \\
\hline Non-Christian & 18 & 58.1 & 40.2 & 40.2 & 0.050 \\
\hline \multicolumn{6}{|l|}{ Occupation } \\
\hline Salaried & 7 & 22.6 & 44 & 23.3 & \\
\hline Unsalaried & 24 & 77.4 & 145 & 76.7 & 0.570 \\
\hline \multicolumn{6}{|l|}{ Education } \\
\hline Formal & 16 & 51.6 & 129 & 68.3 & \\
\hline Informal & 15 & 48.4 & 60 & 31.7 & 0.060 \\
\hline
\end{tabular}

Among the socio-demographic variables, only religion was significantly associated with good practice of use of ITNs (P-value $=0.05)$.

Results of logistic regression analysis: No socio-demographic variable was significantly related to good practice of use of ITNs.

Table 6

Regression analysis of socio-demographic variable against practice of use of ITNs

\begin{tabular}{llllll}
\hline Variable & $\mathrm{B}$ & $\begin{array}{l}\text { Wald's } \\
\text { statistics }\end{array}$ & $\begin{array}{l}\text { Odds } \\
\text { ratio }\end{array}$ & $95 \%$ CI & P-value \\
\hline Age & 0.000 & 0.002 & 1.433 & $-0.180-0.180$ & 0.999 \\
Parity & -0.035 & -0.591 & 0.851 & $-0.153-0.082$ & 0.555 \\
Marital status & -0.138 & -1.266 & 0.413 & $-0.354-0.077$ & 0.207 \\
Religion & 0.074 & 1.454 & 2.059 & $-0.026-0.174$ & 0.148 \\
Occupation & -0.022 & -0.381 & 1.040 & $-0.135-0.091$ & 0.703 \\
Education & 0.074 & 1.349 & 2.016 & $-0.034-0.182$ & 0.179 \\
\hline
\end{tabular}




\section{DISCUSSION}

Knowledge on malaria illness: Malaria was reported as the main health problem by majority of the pregnant women. This was in consistence with trend as seen in the district hospital records and at national reports $(2,3)$.

Nearly all respondents stated that malaria was transmitted through mosquito bite. This contrasts with study findings of Aikins et al in Ghana which found that few of the respondents knew that mosquitoes transmitted malaria (15).

Anaemia and maternal death were the most common complications mentioned which points to the high maternal mortality ratio in the district (3). ITNs use can be used as one strategy to achieve millennium development goals (MDG).

Malaria was considered as a preventable disease to majority of the women by use of mosquito nets. Though few mentioned taking of drug as a preventive measure (metakelfin and fansidar which they routinely buy in shops), they used the drugs for prevention and treatment interchangeably. The two drugs have now been removed as first line drug in treatment of malaria due to high resistance in many regions in Kenya and have been replaced by Artemesine combined therapy (2). The two drugs are currently used for IPT in pregnancy in second and third trimesters.

Knowledge of the protective role of ITNs during pregnancy: Though majority knew the difference between ITNs and ordinary bed nets, some did not. Nearly one in every four women did not know the benefit of ITNs use. This is worrying as there had been Rift Valley fever campaign one year earlier (2006) where ITNs were distributed for free to the community for use in the disease control. Communication on the benefits of ITNs use needs to be a continuous process through advocacy.

Majority of the women mentioned the advantage of ITNs as preventing somebody from getting malaria but some said it prevented mosquito nuisance. This is similar to Klein et al study in Guatemala (16).

Time when ITNs should be used was varied with majority of pregnant women mentioning every night. Some thought ITNs should be used during rainy seasons. Kilifi is a malaria endemic zone where transmission occurs throughout the year with upsurge during rainy seasons. The misconception that ITNs should only be used during rainy season when mosquitoes are assumed to be high is a major drawback in the fight against malaria.

Level of knowledge in relation to socio-demographic variables: The study revealed that adequate level of knowledge on malaria and ITNs was high in women in young age group, those with lower parity, married, Christians, unsalaried and the educated than in older age group, those with high parity, unmarried, salaried and uneducated. There was significant association between education and adequate level of knowledge. There is a big concern as non-Christians formed a big proportion (43\%). There is a need to include religious leaders in the malaria education campaign strategy. The curriculum of the Madrassa and churches may be lacking in terms of malaria prevention methods.

The high level of adequate knowledge among women of lower parity is encouraging since prime-gravidas have a high prevalence of placental parasitaemia and risk of developing severe anaemia than grand multi-parous (4). There is great concern as those with no education constituted a big proportion (34\%). There was significant association between level of education and adequate knowledge. Level of education is paramount in enhancing health knowledge in the community. The current policy of universal primary and secondary education in Kenya is a noble idea of increasing health knowledge in the fight against diseases like malaria.

Attitude ofpregnantwomen on use ofITNs: Good attitude towards use of ITNs among the women was generally low. Attitude had a major influence on ITNs use. Some pregnant women did not sleep under ITN the night before the interview, because it felt hot inside the net. This finding is similar to Yohannes' et al (20). Though there may be some discomfort sleeping under ITNs, the benefits far outweighs the discomfort. The discomfort has been minimised by having mosquito nets made of nylon or other synthetic material, which is lighter; furthermore it has numerous holes that allow ventilation (5). Use of light clothing during hot season is advisable.

Some women did not sleep under the bed net because there were no mosquitoes during that period. This finding is similar to Tsuyuoka et al Zimbabwe (21). Mosquito population may be small just before rainy season, but they are still infectious (5). The decision to buy bed net was personal in majority of women. Some made the decision after advice from health workers (21). The health worker's role in influencing bed net use is paramount and should be encouraged.

In the FGD, women thought the drug in the bed may be meant to control their family size. This finding is similar to other studies in Uganda and Western Kenya $(11,12)$. This misconception could triger stigma in the community. Though the drug may cause minor skin reaction or sneezing, it is safe with no major side effects (5). The best way to fight stigma is through health education with full involvement of the community. 
FGD also revealed that women did not use ITNs since they had not been used to seeing their parents use them. There was no role model to emulate. These findings were similar to Baume's in Ethiopia (10). Poor accessibility of medical information especially deep in the village level may contribute to this scenario.

Attitude of ITNs use in relation to socio-demographic variables: Attitude on ITNs use was not significantly related to any socio-demographic variables. This was surprising in that despite the high level knowledge on malaria, good attitude was low.

Attitude is a complex thing. It cuts across ages, parity, religion, occupation, education and marital status. It createsirrational perspective in people which influence health care service uptake even when it is given free. Changing people's attitude is difficult and takes time. Many methods needs to be applied and when they fail, different ones should be tried. People needs to be motivated and encouraged to adopt ITNs use. Attitude on ITNs remains a big challenge. ITNs campaigns should create environment thatencourage change. This is by allowing people to be involved and giving them opportunity to talk about their issues freely.

Practice of ITNs use in prevention of malaria during pregnancy: Majority of pregnant women said they used ITNs to prevent malaria while ordinary bed nets were used by only a few. These findings are similar to Baume's study in Senegal, Nigeria, Zambia, Ghana and Ethiopia (10). ITNs are more effective than ordinary bed nets and do not need re-treatment every six months.

The study found out a high percentage use of ITNs $(70.5 \%)$ which is very encouraging. This finding is similar to Noor's et al study in Kenya (23). High ITNs coverage has advantage of the 'mass effect' on the local mosquito population when they are used on a community-wide basis (7). If this high coverage could be sustained and extended to other vulnerable groups of the population, universal coverage is possible and this would lead to drop in the malaria cases that are prevalent in the district.

Majority of women reported using ITNs every night while some used ITNs during rainy season. This practice of using ITNs only during rainy seasons should be discouraged as malaria transmission is perennial in Kilifi. Intermittent use of ITNs reduces its effectiveness.

The study found out that over $55 \%$ had had the net for a short period of 11 months. Majority got free bed nets during the campaign against Rift Valley fever in 2006. This implies that free ITNs distribution increases coverage as well as use.
This should be adopted as strategy to increase net coverage and use. These findings are similar to Guyatt's and Noor's studies in Kenya $(8,23)$. The study also shows that ITNs campaigns increased the coverage and usage.

In the FGD poverty was cited as a reason why pregnant women did not use ITNs. This finding is similar to Aikins' in five West African communities (14). Subsidizing increases bed net coverage but giving free bed nets has a higher coverage and use especially among the poor groups as demonstrated by Noor's et al (23).

Weather influenced ITNs use. Some women avoided using ITNs during hot weather sighting discomfort. This finding is similar to Laii et al Western Kenya (18). Malaria transmission is perennial in Kilifi. Design of houses to allow proper ventilation is needed and the houses should have window mesh that prevents mosquito entry into the houses. People should be advised to use light clothing to minimise discomfort.

Practice of use of ITNs in relation to socio-demographic variables: Good practice on use of ITNs was generally high. Good practice on use of ITNs was high among those with lower parity. The study results are encouraging as the group was the majority. This is important as the prime-gravidaes and those with lower parity have high prevalence of malaria parasitaemia than grand multiparous. Similarly, they are likely to suffer from severe malaria complications especially anaemia than grand multiparous (4).

Good practice on use of ITNs was high among the Christians. There was significant association between religion and good practice. The low level of good practice in the non-Christians is of great concern as they formed a big proportion (43\%). This study brings into focus on the curriculum of madrasas, churches and Sunday schools in terms of malaria prevention. This would be a good forum to dissimilate message more so because Muslim and church leaders act as role model in the community.

Good practice was high in the educated than non-educated but interestingly education was not significantly associated with good practice. These findings are similar to Aikins' study in Gambia (15). The challenge is to put this knowledge into good practice of ITNs use. This implies that different socio-cultural factors must be considered when behavioural interventions for malaria control are designed and implemented.

\section{CONCLUSION}

Malaria was identified as a serious heath issue with childrenbelow fiveyears and pregnant women mostat 
risk. Malaria was considered as a preventable disease in pregnancy by use of ITNs to avoid anaemia and maternal deaths which are prevalent in the district.

The knowledge of the protective role of ITNs during pregnancy was high though there was some knowledge deficiency particularly on time to use them that was influenced by rainy season, hot weather, and assumption of decreased mosquito density at home. ITNs were more used than ordinary bed nets. The use of ITNs was high surpassing the Abuja target of $60 \%$. The main challenge is to sustain this high coverage and more so in the interior parts of the district where accessibility, acceptability and affordability may be an issue. Weather influenced the ITNs use with more people using them during cooler and rainy season while fewer people used them during hot season. Culture had a major impact on ITNs use. There still remains misconception and stigma on ITNs use among women in the community. There is fear the high coverage my not be sustained unless the major misconceptions are addressed through targeted health education. Poverty still remains a barrier to ITNs ownership and use to many women in the community. Giving free bed nets to pregnant women increases their usage and this should be regarded as an important delivery system in increasing access to and use of ITNs among vulnerable groups.

Women attitude had a major influence on ITNs use. The belief that sleeping under ITNs is uncomfortable due to the perceived warming effect of the net reduced their use. The belief that there are no mosquitoes at certain times of the year or the numbers may be low affected the ITNs use. The misconception that the insecticide in the bed net may be there as a family planning method created fear which made women not only refuse to use bed net but returned them despite getting them for free. Colour and shape of the bed had different cultural interpretations which influenced the bed net use. Blue or green conical bed nets were more preferred than white / rectangular ones.

Use of ITNs has not been fully internalised especially in the interior part of district where young women grew up without seeing their parents use them. The usage in these areas may remain low as culture takes time to change unless programmes to accelerate practice are put in place. The attitude towards ITNs use among the pregnant women was an issue. Good attitude towards use of ITNs was generally low. Attitudes tend to create irrational decisions which influence health care service uptake even when it is given for free.

\section{ACKNOWLEDGEMENTS}

To the people of Kilifi district, who participated in the study and the staff of Ministry of Health at
Chesimba, Vipingo, Vitengeni, Bamba, Ganze health centres and Kilifi district hospital. More thanks goes to Miss Salama and Christine, the field clerks, for doing a good job of data collection. The thesis was submitted as part fulfilment for the award of degree of Masters of Public Health of the University of Nairobi in the year 2008.

\section{REFERENCES}

1. World Health Organization's World Malaria Report 2005.

2. Republic of Kenya Ministry of Health, National Malaria Strategy, 2001.

3. Kilifi district annual report 2006/2007.

4. Shulman, C.E., Marshall, T., Dorman,E.K., etal. Malaria in pregnancy: adverse effects on haemoglobin levels and birth weight in primigravidae and multigravidae. Trop. Med. Inter. Hlth. J. 2001; 6: 770-778.

5. Gilles, H.M. and Warrell, D.A. Bruce-chwatt'S Essential malariology, 1993 Textbook, pg 114, Third Edition.

6. Lengeler, C., Jacqueline, C. and Don de Savingny. Net gain. A new method for preventing malaria deaths, 1996 Textbook, pp 32, First Edition.

7. Soremekun, S., Maxwell, C., Zuwakuu, M.,-et al. Measuring the efficacy of insecticide treated bed nets: the use of DNA fingerprinting to increase the accuracy of personal protection estimates in Tanzania. Trop. Med. Inter. Hlth. 2004; 9: 664.

8. Guyatt, H. and Ochola, S. Use of bed nets given free to pregnant women in Kenya. Lancet. 2003; 362 (9395):1515-1516.

9. Lin, K., Aung, S., Lwin, S., etal. Promotion of insecticidetreated mosquito nets in Myanmar. Southeast Asian J. Trop. Med. Publ. Hlth. 2000; 31: 444-447.

10. Baume, C. Understanding mosquito net use at the household level: Are household mosquito nets being used? If so, Who uses them? Net mark 2004 Survey on Insecticide-Treated.

11. Mbonye, K.A., Neema, S. and Magnussen, P. Preventing malaria in pregnancy: a study of perceptions and policy implications in Mukono district, Uganda. Oxford J. Hlth Policy Plann. 2005; 21: $17-26$.

12. Alaii, J.A., van den Borne, H.W., Kachur, S.P., et al. Perceptions of bed nets and malaria prevention before and after a randomized controlled trial of permethrintreated bed nets in western Kenya. Amer. J. Trop. Med. Hyg. 2003; 68(4 Suppl):142-148.

13. Agyepong, I. A. Malaria: ethno medical perceptions and practice in an Adangbe farming community and implications for control. Soc. Sci. Med. J. 1992; 35: 131-137.

14. Aikins, M.K., Pickering, H. and Greenwood, B.M. Attitudes to malaria, traditional practices and bed nets (mosquito nets) as vector control measures: a comparative study in five western African countries. J. Trop. Med. Hyg. 1994; 97: 81-86.

15. Aikins, M.K., Pickering, H., Alonso, P.L., et al. A malaria control trial using insecticide-treated bed nets and targeted chemoprophylaxis in a rural area of the Gambia, Perceptions of the causes of 
malaria and of its treatment and prevention in the study area. Trans. Royal Society Trop. Med. Hyg. 1993; 87 (Suppl 2): 25-30.

16. Klein, R.E., Weller, S.C., Zeissig, R., et al. _Knowledge, beliefs, and practices in relation to malaria transmission and vector control in Guatemala. Amer. J. Trop. Med. Hyg. 1995; 52:383-388

17. Rodriguez, A.D., Penilla, R.P. and HenryRodriguez, M. Knowledge and beliefs about malaria transmission and practices for vector control in southern Mexico. Salud Publica de Mexico 2003; 45:110-116.

18. Laii, A.J., Awley, W., Kolczak, S.M., et al. Factors affecting use of permethrin-treated bed nets during a randomized controlled trial in western Kenya. Amer. J. Trop. Med. Hyg. 2003; 68(4 suppl): 137-141.

19. Okrah, J., Traore, C., Pale, A., et al. Community factors associated with malaria prevention by mosquito nets: an exploratory study in rural Burkina Faso. Trop. Med. Inter. Hlth. J. 2002; 7: 240-248.

20. Yohannes, K., Dulhunty, J.M., Kourleoutov, C., et al. Malaria control in central Malaita, Solomon Islands. The use of insecticide-impregnated bed nets. Acta Tropica. 2000; 75: 173-183.

21. Tsuyuoka, R., Midzi, S.M., Dziva, P. and Makunike, B. The acceptability of insecticide treated mosquito nets among community members in Zimbabwe. Central Afr. J. Med. 2002; 48: 87-91.

22. Kenya Demographic Health Survey (KDHS 2003).

23. Noor, M.A., Amin, A. A., Akhwale, S.W and Snow, W.R. Increasing coverage and decreasing inequity in insecticide-treated bed net use among rural Kenyan children. PLoS Med. J. 2007; 4: 255. 